\title{
Early versus late treatment of voiding dysfunction with pelvic neuromodulation
}

\author{
Magdy M. Hassouna, MD, PhD; Mohamed S. Elkelini, MD
}

See related article on page 111

\begin{abstract}
Introduction: Pelvic neuromodulation is an established method of treating voiding dysfunction. Little is known about the pathophysiology associated with voiding dysfunction. Reports have suggested that a delay in treating patients with sacral neuromodulation therapy can impact the success rate of this type of treatment in voiding dysfunction. We examined patient response to pelvic neuromodulation when it was applied early versus late in the postdiagnosis of voiding dysfunction.
\end{abstract}

Methods: We conducted a retrospective study of 42 patients (38 women and 4 men) with voiding dysfunction who underwent surgery for implant with the Interstim (Medtronic, Minneapolis, Minn.). Prior to implantation, patients were required to pass a percutaneous nerve evaluation (PNE) over a 1-week period. Patients were observed for 20-48 months postimplantation. All patients recorded their voiding parameters at baseline, after screening and every 6 months thereafter. Twenty patients (in the early group) underwent implant surgery with the neurostimulator 2-4 weeks post-PNE, and 22 patients (the late group) had the device implanted 6-24 months post-PNE owing to local logistical circumstances.

Results: In the early group, 16 of 20 patients (80\%) maintained a good response. In the late group, 13 of 22 (59\%) patients showed a good response. Groups were well matched in terms of age, duration of voiding dysfunction and incidence of comorbidity.

Conclusion: Patients who were delayed more than 6 months in receiving the neurostimulator implant showed a worse response than did patients who had the device implanted soon after PNE. This indicates the possibility of disease progression, which may limit the response to sacral neuromodulation.

CUAJ 2007;1(2):106-10

S acral neuromodulation (SNM), using permanent foramen S3 electrode, offers an alternative treatment for patients with conditions refractory to conventional measures. SNM has been approved by the US Food and Drug Administration for 3 indications: urge incontinence (UI), urge frequency (UF) and nonobstructive urinary retention. Several reports have been published regarding the efficacy of SNM in the treatment of UI, UF and nonobstructive urinary retention. ${ }^{1-4}$

SNM is considered a minimally invasive procedure, and when compared with the more drastic procedures to control intractable over- active bladder symptoms, SNM has fewer complications and has provided patients with more durable and consistent bladder control over time.

The purpose of the current study is to determine whether a delay in SNM can affect the long-term outcome of treatment in patients with voiding dysfunction.

\section{Methods}

In this retrospective study, 42 patients (38 women and 4 men) with voiding dysfunction (28 with UF, 8 with nonobstructive urinary retention and 6 with $\mathrm{UI}$ ) were selected for SNM based on successful percutaneous nerve evaluation (PNE) of the sacral nerve roots. Treatment and follow-up of patients occurred between 2000 and 2004 at the University Health Network. Prior to the implantation of a neurostimulator (Interstim, Medtronic, Minneapolis, Minn.), patients had to pass the PNE screening test that showed > 50\% improvement in one or more voiding parameters (e.g., volume per void, number of daily voids, number of pads used daily, postresidual volume and number of daily catheterizations) over a 1-week period (Fig. 1). The study protocol for screening using PNE and SNM has been reported. ${ }^{5,6}$ Patients were stratified into 2 groups, early versus late, based on whether they received the implantable neurostimulator early or later after the PNE. We obtained research ethics approval for this retrospective study. All patients were operated on by a single surgeon. The implantable device was Interstim model 3023, which was located in a subcutaneous pouch in the buttock. The extension cable measured $10 \mathrm{~cm}$ (Medtronic Inc., model 3095-10). The permanent quadripolar electrode model 3080 was used in 12 and 
14 patients in the early and late groups, respectively. The rest of the patients received the "tined" electrode lead model 3889. We used only hardware manufactured by Medtronic Inc.

We verified the duration of the voiding dysfunction in both groups as well as the comorbidity and the medications the patients were taking.

All patients were observed every 6 months for 24-48 months, with a mean of 32.5 months. In each follow-up visit, the patients had to present a 4-5 day voiding diary recording their voiding parameters (number of voids per day, volume in $\mathrm{mL}$ per void and urine volume obtained by catheter) and incontinence (number of pads used daily and amount of daily leak). The parameters in the voiding diaries were compared individually with the baseline and with the postscreening (post-PNE diary). To qualify for the implantation of a permanent neurostimulator, the patient had to show an improvement of at least $50 \%$ in one or more of the voiding parameters mentioned in the post-PNE diary when compared with the baseline diary. The criteria of success (i.e., responders) in the postoperative follow-up were voiding parameters similar to or better than that shown on the post-PNE diary and overall patient satisfaction. The criteria of failure (i.e., nonresponders) were voiding parameters worse than post-PNE diary but better than baseline diary, voiding parameter returning to the baseline diary and the removal (i.e., explantation) of the neurostimulator due to lack of efficiency, complications or both.

The voiding parameters were subject to a statistical program (SigmaStat 3.0A). We compared data collected from voiding diaries using 1-way analysis of variance followed by a post-hoc comparison of the means; the significant difference was set at $p<0.05$.

\section{Results}

The results are presented as a comparison between the voiding parameters recorded in the diaries of patients in the early and late implantation groups.

The early group included 20 patients (18 women and 2 men) who received the permanent implant 2-3 weeks after showing a successful screening test. Patients in the early group had voiding dysfunction for 20-60 months (mean $34 \mathrm{mo}$ ).

The late group included 22 patients ( 20 women and 2 men) who received the implant 6-24 months (mean $18.5 \mathrm{mo}$ ) after the screening test. The dura- tion of the voiding dysfunction was 19-70 months, with a mean of 30 months duration. There was no statistical difference in the duration of voiding dysfunction between the 2 groups $(p>0.05)$. The patients' ages were similar in the 2 groups, averaging 42 years in the early group and 45 years in the late group. The intake of the medication and the comorbidity in both groups were very similar.

In the early group, 16 patients were diagnosed with UF with suprapubic discomfort or pain, compared with 12 patients in the late group. There were 2 patients with urinary retention in the absence of evidence of outlet obstruction in the early group, whereas there were 6 in the late group. Two patients in the early group and 4 in the late group had UI (Table 1).

\section{Screening test (PNE)}

There were 12 PNE attempts performed for 12 patients in the early group. For the late group,

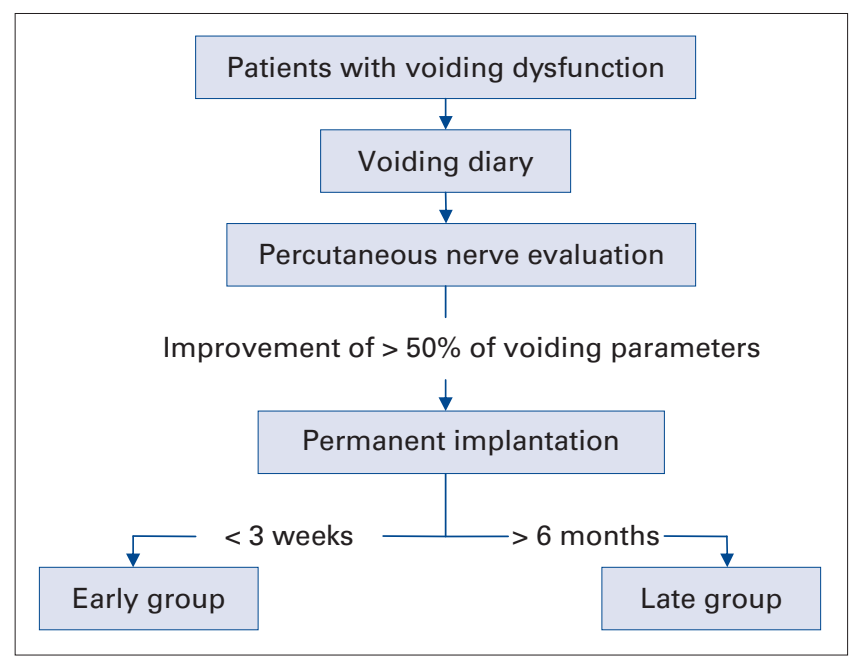

Fig. 1. Algorithm showing the distribution of 42 patients into the early and late groups.

Table 1: Distribution of the 42 patients in both groups according to cause of voiding dysfunction

\begin{tabular}{|c|c|c|c|c|}
\hline \multirow{3}{*}{$\begin{array}{l}\text { Voiding } \\
\text { dysfunction }\end{array}$} & \multicolumn{4}{|c|}{ Group; no. of patients } \\
\hline & \multicolumn{2}{|c|}{ Early } & \multicolumn{2}{|c|}{ Late } \\
\hline & $\mathrm{R}$ & NR & $\mathrm{R}$ & NR \\
\hline Urge frequency & 14 & 2 & 8 & 4 \\
\hline Urge incontinence & 1 & 1 & 2 & 2 \\
\hline Urinary retention & 2 & 0 & 4 & 2 \\
\hline \multicolumn{5}{|c|}{$\mathrm{R}=$ responders; $\mathrm{NR}=$ nonresponders } \\
\hline
\end{tabular}


24 PNE attempts were performed for 22 patients. Two patients had their PNE repeated owing to technical difficulties.

\section{Waiting time}

In the early group, all 20 patients had their permanent implant within 2-3 weeks. In the late group, patients had to wait for 6-24 months, with a mean waiting time of 18 months.

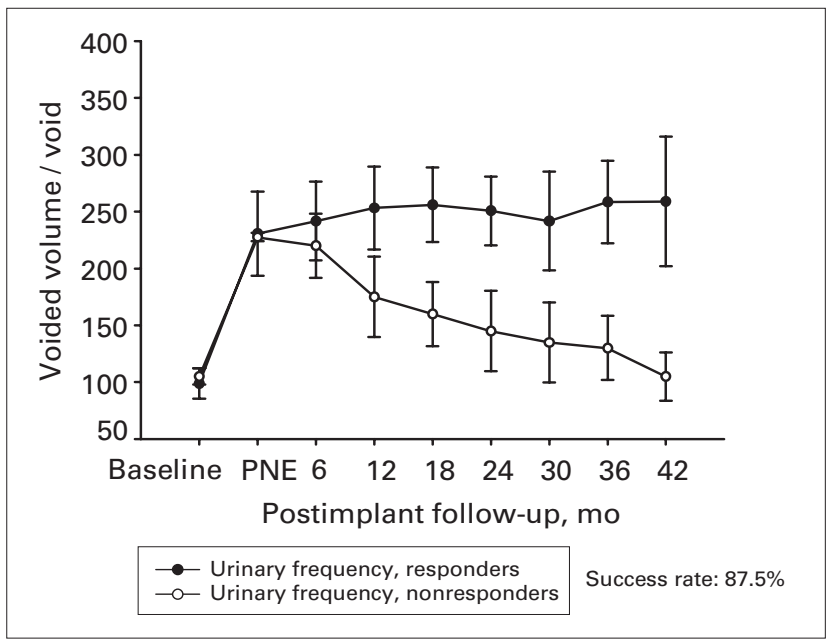

Fig. 2. Voided volume per void of patients with urge frequency in the early group $(n=16)$. PNE $=$ Percutaneous nerve evaluation.

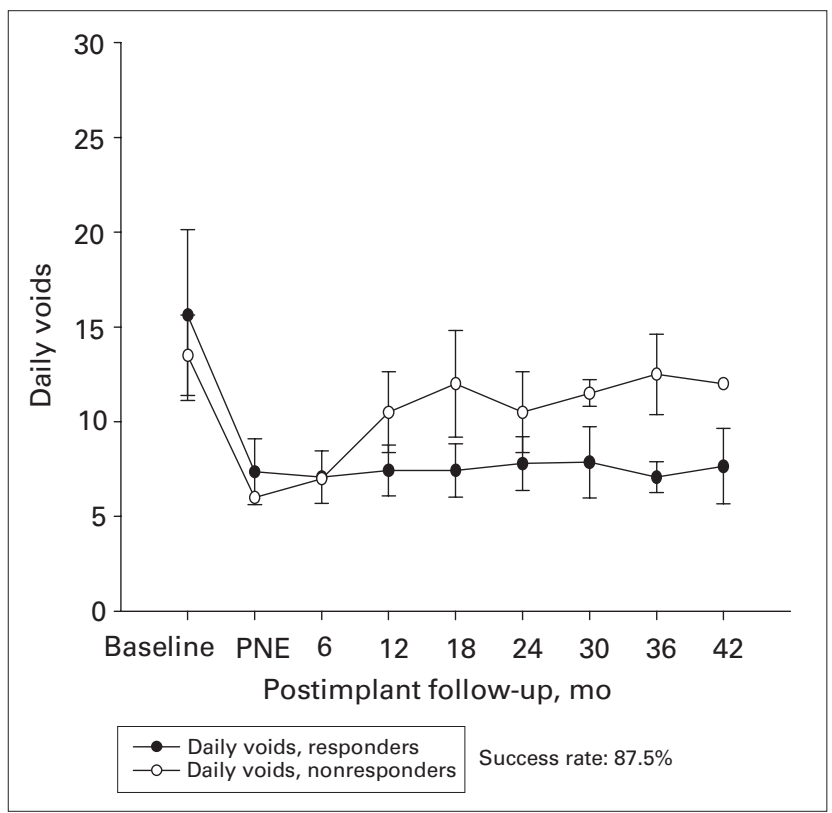

Fig. 3. Number of daily voids of patients with urge frequency in the early group $(n=16)$. PNE $=$ Percutaneous nerve evaluation.

\section{Location of the permanent electrode}

All patients had a single electrode (model 3889 Medtronic Inc.) implanted. Of patients, 25 had the implantation of a permanent neurostimulator on the left third sacral nerve root, and 15 patients had it on the right third sacral root. In 2 patients, the permanent electrode was implanted on the right fourth sacral nerve root.

\section{Response during the follow-up}

In the early group, 17 of 20 patients (85\%) showed good response in terms of their symptoms and their overall satisfaction for a follow-up period of 32.5 (mean) months. Among the subgroup of patients with UF, 14 of 16 patients had a good response $(87 \%)$ both in the voided volume per void and the number of daily voids (Fig. 2 and Fig. 3). The improvement in the voiding parameters was maintained through the follow-up period, as depicted in Figure 2. Three patients had poor response; 1 was due to wound infection, and the other 2 showed poor response 8 and 12 months after the implantation of the permanent stimulator, respectively.

In the late group, 14 of 22 patients (63.6\%) showed good response in their symptoms and their overall satisfaction. Six of the 22 patients had worse symptoms than those reported in most postscreening diaries; however, 6 months after the implantation of the permanent stimulator, their symptoms were still better than those reported in the baseline diaries. Figure 4 depicts the responses of patients in the subgroup with UF. Two patients were excluded because of lack of efficiency after 6-12 months (mean 9 mo). The mean time between the implantation and the onset of the poor response was 9 months. The patients in this group who showed an improvement in the voiding parameters maintained their response through the follow-up period. (Fig. 4 and Fig. 5)

\section{Discussion}

Pelvic neuromodulation has been an established modality of treatment for voiding dysfunction for the past 10 years. Results of several recent studies have shown level 3 evidence that SNM benefits patients with UI, UF and urinary retention. The discrepancy in the success rates, as pointed out in 
the literature, can be attributed to change in the technique, the disease progression or both, leading to voiding dysfunction.

The current study addresses an important issue - the time factor between the screening of the patient and the implantation of a permanent device. Over the past 7 years, the Division of Urology at the Toronto Western Hospital has been a centre of referral for all patients with voiding dysfunction throughout Ontario. Those patients are referred because of intractable voiding dysfunction and because they did not show a satisfactory response to conventional therapy. Patients were screened according to the protocol that has been previously described.? In short, patients were asked to fill out a voiding diary to record voiding parameters. The patients were then screened using a PNE for the sacral nerve roots bilaterally to test the integrity of the sacral nerve roots. The nerve root that showed a good response (motor, sensory or both) was chosen for stimulation via a temporary electrode. The patients were sent home with a temporar electrode connected to an external pulse generator for 4-7 days. During that time, the patient was asked to complete a second voiding diary. Those patients who showed a 50\% improvement in one or more of the voiding parameters, as recorded in the second voiding diary, were scheduled to have a permanent device implanted. The location of the temporary electrode was recorded through an x-ray film of the sacrum in the lateral and antero-posterior views. Those films were used as a guide during the implantation of the permanent electrode. All patients were followed up every 6 months, using a voiding diary to record their postoperative response to the implanted device.

The discrepancy in the waiting period between the 2 groups of patients is owing to local circumstances. Due to budget constraints, there has been a ceiling of 12 set on the number of annual implantations. Because the Toronto Western Hospital was the only centre in Ontario offering the Intrestim therapy for voiding dysfunction, we had to accept all patients referred from the entire province. Because 1 of 3 patients screened with PNE ended up having a permanent implant, those patients who were qualified for the permanent implant had to wait for up to 24 months, owing to budget restrictions, before receiving the permanent device. Conversely, patients referred from outside Ontario had the screening test and implantation of the per- manent stimulator within a few weeks. Owing to logistic arrangements, those patients did not have to wait for the extended period of time, because their respective provincial health insurance plans were paying for the devices.

The discrepancy in the outcome of the Interstim therapy favoured better response in the early group of patients (85\% compared with $63.6 \%$ in the late group). Once patients responded to the SNM, this response was maintained during follow-up, irrespective of whether the patient received treatment

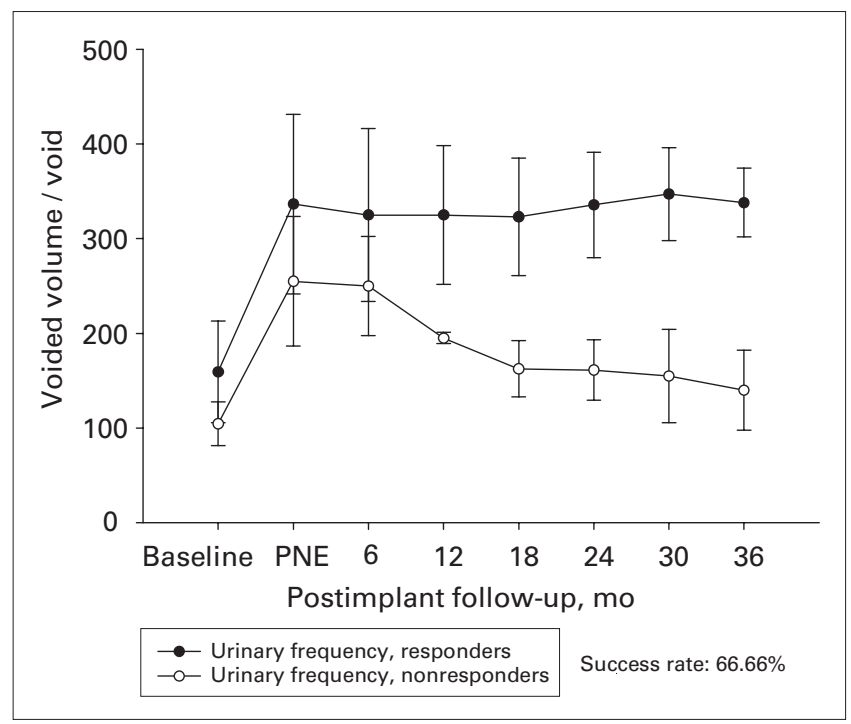

Fig. 4. Voided volume per void of patients with urge frequency in the late group $(n=12)$. PNE $=$ Percutaneous nerve evaluation.

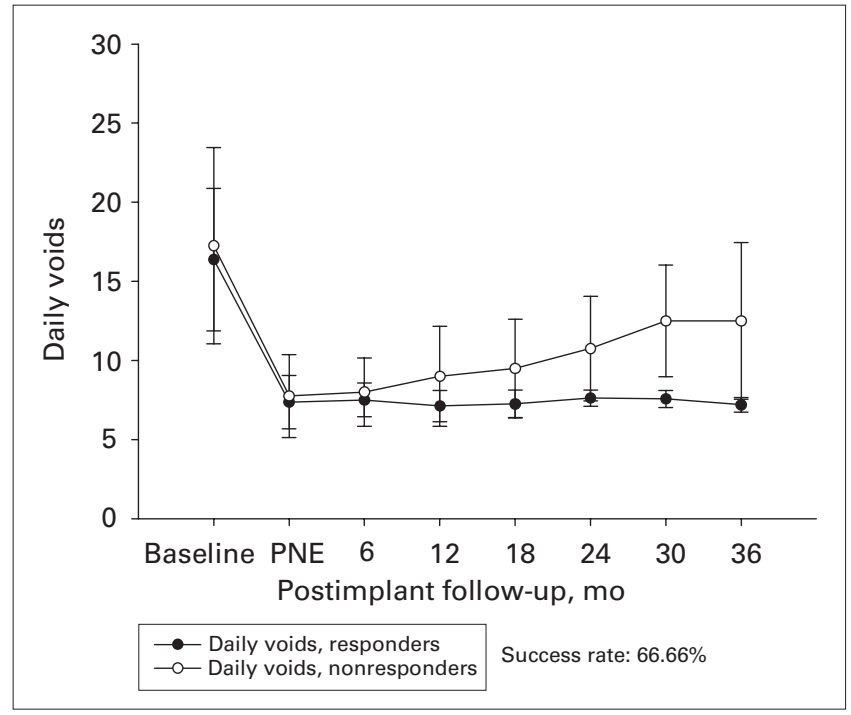

Fig. 5. Number of daily voids of patients with urge frequency in the late group $(n=12)$. PNE $=$ Percutaneous nerve evaluation. 
Hassouna and Elkelini

early or late after the PNE. Upon comparison, these 2 groups of patients had similar disease duration (mean 34 months in the early group v. 32 months in the late group). The patients' ages were similar in the 2 groups. The 2 groups also showed similarity in comorbidities, such as hypertension disease and diabetes. They were also similar in terms of medications. None of the poor outcomes in either group could be attributed to a technical reason in the hardware since the latter was implanted equally in the patients of both groups. The lack of efficacy of the SNM could be attributed to the emergence of such concomitant diseases as diabetes mellitus or cereberovascular accidents. Those diseases have a negative impact on bladder function even after SNM. The patients in the 2 groups were very similar in terms of diagnosis of voiding dysfunction, age group, comorbidity and medication intake; the only difference was the latent period before the implantation of the permanent stimulator. Moreover, we did not find any difference in the time taken to the onset of poor response in either group (mean $9 \mathrm{mo}$ ). In clinical practice, the trend has been to offer the SNM to the patient after the failure of either treatment with pharmacotherapy, pelvic floor rehabilitation or both to control the voiding dysfunction. A study from The Netherlands showed that patients who present with voiding dysfunction that lasted a short period of time had a higher chance of a successful outcome after test stimulation, compared with patients who had dysfunction for a relatively longer period of time. ${ }^{8}$

We strongly believe that the pathological process causing the voiding dysfunction is progressive, because the only difference in the 2 groups in the current study was the patients' wait time for the permanent implant. If voiding dysfunction remains untreated for a long time, it makes it more difficult for SNM to restore the normal balance between the afferent and the efferent components of the sacral reflex arc. The current study does not address the critical time beyond which SNM therapy may fail to restore or revert the voiding function. Studies have shown that the development of aberrant spinal reflexes are the basis of neuroplasticity. ${ }^{9,10}$ Spinal neuroplasticity could be responsible for the high failure rate in the patients who had to wait for the permanent implant. Currently, we recommend the rescreening of the patients who had to wait for 6 months to note whether they still show an appropriate response. Staged implants would be another alternative to introduce before the implantation of the permanent device.

Division of Urology, Toronto Western Hospital, University Health Network, University of Toronto, Toronto, Ont.

Acknowlegements: This work was supported by the Department of Surgery at the University Health Network, University of Toronto, Toronto, Ont.

This article has been peer reviewed.

Competing interests: None declared.

\section{References}

1. Schmidt RA, Jonas U, Oleson KA, et al. Sacral nerve stimulation for treatment of refractory urinary urge incontinence. Sacral Nerve Stimulation Study Group. J Urol 1999;162:352-7.

2. Hassouna MM, Siegel S, Nyeholt AA, et al. Sacral neuromodulation in the treatment of urgency-frequency symptoms: a multicenter study on efficacy and safety. J Urol 2000;163:1849-54.

3. Jonas $U$, Fowler $C J$, Chancellor $M B$, et al. Efficacy of sacral nerve stimulation for urinary retention: results 18 months after implantation. J Urol 2001;165:15-9.

4. Siegel SW, Catanzaro F, Dijkema HE, et al. Long-term results of a multicenter study on sacral nerve stimulation for treatment of urinary urge incontinence, urgency-frequency, and retention. Urology 2000;56(Suppl 1):87-91.

5. Shaker $\mathrm{HS}$, Hassouna M. Sacral nerve root neuromodulation: effective treatment for refractory urge incontinence. J Urol 1998;159:1516-9.

6. Shaker HS, Hassouna M. Sacral root neuromodulation in idiopathic nonobstructive chronic urinary retention. J Urol 1998; 159:1476-8.

7. Elabbady AA, Hassouna MM, Elhilali MM. Neural stimulation for chronic voiding dysfunctions. J Urol 1994;152:2076.

8. Scheepens WA, Van Koeveringe GA, De Bie RA, et al. Long-term efficacy and safety results of the two-stage implantation technique in sacral neuromodulation. BJU Int 2002;90:840-5.

9. de Groat WC. A neurologic basis for the overactive bladder. Urology. 1997;50 (6A Suppl):36-52; discussion 53-6.

10. de Groat WC. Plasticity of bladder reflex pathways during postnatal development. Physiol Behav 2002;77:689-92

Correspondance: Dr. Magdy M. Hassouna, Toronto Western Hospital, MP 8-206, 399 Bathurst Street, Toronto ON M5T 2S8; fax 416 603-1961; mhassouna@yahoo.com 